\title{
Switchable photonic crystal cavity by liquid crystal infiltration
}

Pierre-Yves Baroni

Qing Tan

Vincent Paeder

Armando Cosentino

Matthieu Roussey

matthieu.roussey@epfl.ch

Toralf Scharf

Hans Peter Herzig

Wataru Nakagawa
Ecole Polytechnique Fédérale de Lausanne, Institut de Microtechnique, Optics \& Photonics Technology Laboratory, Breguet 2, 2000 Neuchâtel, Switzerland

Ecole Polytechnique Fédérale de Lausanne, Institut de Microtechnique, Optics \& Photonics Technology Laboratory, Breguet 2, 2000 Neuchâtel, Switzerland

Ecole Polytechnique Fédérale de Lausanne, Institut de Microtechnique, Optics \& Photonics Technology Laboratory, Breguet 2, 2000 Neuchâtel, Switzerland

Ecole Polytechnique Fédérale de Lausanne, Institut de Microtechnique, Optics \& Photonics Technology Laboratory, Breguet 2, 2000 Neuchâtel, Switzerland

Ecole Polytechnique Fédérale de Lausanne, Institut de Microtechnique, Optics \& Photonics Technology Laboratory, Breguet 2, 2000 Neuchâtel, Switzerland

Ecole Polytechnique Fédérale de Lausanne, Institut de Microtechnique, Optics \& Photonics Technology Laboratory, Breguet 2, 2000 Neuchâtel, Switzerland

Ecole Polytechnique Fédérale de Lausanne, Institut de Microtechnique, Optics \& Photonics Technology Laboratory, Breguet 2, 2000 Neuchâtel, Switzerland

Montana State University, Electrical and Computer Engineering Department, Bozeman, MT 597173780 , USA

We report on the fabrication and optical characterization of a switchable photonic crystal nanocavity actuated by liquid crystals. This device acts as a filter presenting a transmission peak around the telecom wavelength $\lambda=1550 \mathrm{~nm}$. Passing from the isotropic to the anisotropic (oriented crystals) state of the liquid crystals, a shift of $\Delta \lambda=13 \mathrm{~nm}$ has been measured, which confirms the theoretical predictions obtained by finite difference time domain simulations. We have developed a photonic crystal nanocavity that can be tuned thanks to the properties of liquid crystals infiltrated in the holes of the photonic device. [D0I: 10.2971/je0s.2010.10057]

Keywords: photonics, nanostructure, photonic crystal, liquid crystal

\section{INTRODUCTION}

The discovery of photonic crystals (PhCs) in 1987, by Yablonovitch and John [1, 2], has opened the way to the miniaturization of optical elements. Widely used as filters [3] thanks to the band gap effect that PhCs present, they are, nowadays, at the heart of large range of integrated optical components such as modulators [4]-[6], switches [7], and superprisms [8]-[10] for multiplexing and demultiplexing for example. At the same time, the tunability of these devices appears as a challenge and different techniques have been used in order to make active photonic crystals, such as acousto [11] or electro-optic [12] effects, nonlinear properties of the materials or thermal [13] effects. Tuning resonant peak by modifying the cavity hole geometry and infiltration of liquid crystals has already been observed [14]. Nevertheless, experimental demonstration of tunable cavities using LC's properties are still rare $[15,16]$.

In this paper we present a switchable photonic crystal filter where the liquid crystal (5CB from Merck) is infiltrated inside the nanostructure [17] to obtain tunability of the optical characteristics. At room temperature the liquid crystal is in its ordered phase and shows anisotropy. The orientation at the sili- con surface is preferentially parallel to the surface [18]. Above the phase transition temperature for $5 \mathrm{CB}$ at $35.5^{\circ} \mathrm{C}$ the liquid crystal is isotropic. Thus, when changing the phase of the liquid crystal, a change in order, anisotropy and refractive index is observed. If the liquid crystal infiltration is sufficient we can act on the photonic bandgap properties of the device by changing the temperature.

The filter is obtained by placing a nanocavity in the center of a photonic crystal waveguide. The aim of the study is to shift the transmission peak generated by the nanocavity by changing the refractive index and the state of liquid crystal infiltrated in the sample.

\section{DESIGN AND FABRICATION}

The basis for the design of the structure studied in this paper is presented in Figure 1(a) and (b). The device consists of a PhC waveguide in a freestanding silicon membrane. A nanocavity is inserted in the center of the guide. The purpose of this study is to characterize the behavior of this nanocavity 


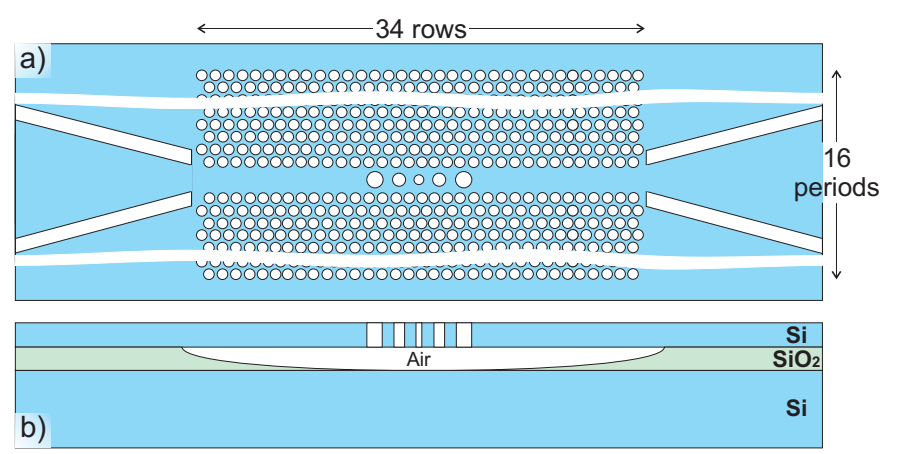

c) cavity type 1

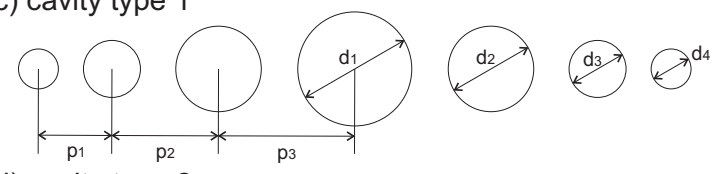

d) cavity type 2

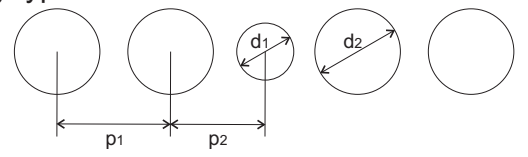

FIG. 1 Nanostructure designs: a) top view, b) cross section along the light propagation, at the center of the waveguide, $c$ ) and d) cavity design and parameters for the two types of nanocavity, without and with liquid crystals respectively.

\begin{tabular}{|c|c|c|c|}
\hline & Cavity type & 1 & 2 \\
\hline \hline Periods & $p_{1}(\mathrm{~nm})$ & 360 & 380 \\
& $p_{2}(\mathrm{~nm})$ & 360 & 470 \\
& $p_{3}(\mathrm{~nm})$ & 482 & - \\
\hline Diameters & $d_{1}(\mathrm{~nm})$ & 290 & 220 \\
& $d_{2}(\mathrm{~nm})$ & 260 & 260 \\
& $d_{3}(\mathrm{~nm})$ & 200 & - \\
& $d_{4}(\mathrm{~nm})$ & 170 & - \\
\hline PhC & $p_{P h C}(\mathrm{~nm})$ & 450 & 430 \\
parameters & $d_{P h C}(\mathrm{~nm})$ & 300 & 320 \\
\hline
\end{tabular}

TABLE 1 Parameters of the two types of nanocavities.

depending on the material infiltrating the holes of the cavity (liquid crystal (LC) or air).

Devices are fabricated on silicon on insulator (SOI) wafers. The thickness of the top silicon ( $\mathrm{Si}$ ) membrane is $340 \mathrm{~nm}$. The photonic crystal is composed of 34 rows of holes in the propagation direction and 16 periods in the transverse direction. The photonic waveguide consists of 1 row of missing holes. The hole diameter is $d=300 \mathrm{~nm}$ and the period is $p=450 \mathrm{~nm}$ (see Table 1). Light reaches the photonic waveguide using a taper from $10 \mu \mathrm{m}$ at the input of the sample to $580 \mathrm{~nm}$ at the input of the photonic waveguide. Note that the silicon oxyde $\mathrm{SiO}_{2}$ has been removed from under the photonic crystal layer, in order to create a free standing membrane of Si. Using a membrane eases the infiltration of liquid crystals into the photonic structure.

Two types of nanocavities have been studied. They are shown in Figure 1(c) and (d) and the parameters are listed in Table 1. The first one, composed of 7 holes, is designed to work when surrounded by air (without liquid crystals) and the second one, composed of 5 holes, works with infiltrated liquid crystal.

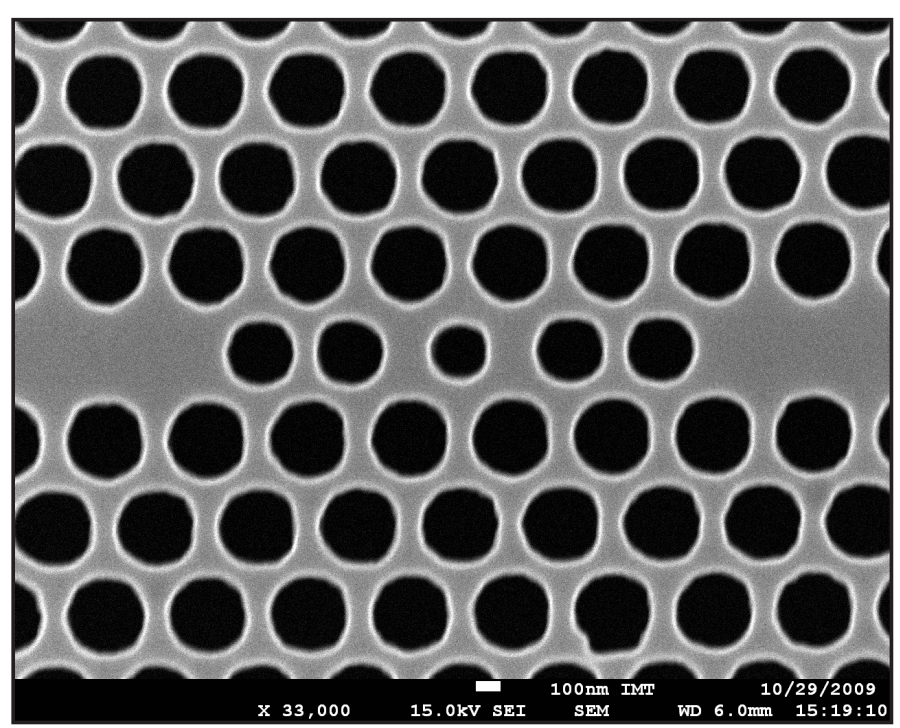

FIG. 2 SEM image of fabricated nanocavity type 2 .

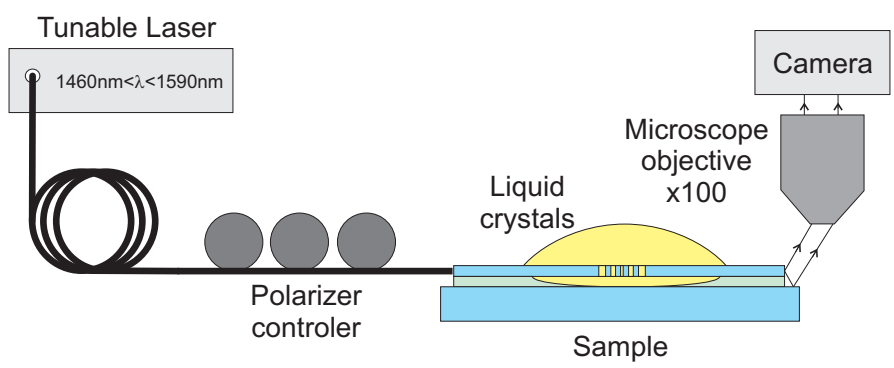

FIG. 3 Scheme of the setup used for the characterization.

The samples are fabricated using e-beam lithography and the membrane is formed by etching in hydrofluoric acid $(H F)$. The etching of the holes has been performed by Inductively Coupled Plasma Reactive Ion Etching (ICP-RIE). The SEM pictures presented in Figure 2 show the fabricated cavity of type 2.

\section{NANOCAVITY CHARACTERIZATION}

The characterization setup is described in Figure 3. A fibered tunable laser (1460 nm $<\lambda<1590 \mathrm{~nm}$ ) is used as a light source (Hewlett Packard Tunable laser source 8168F). Light passes through a fiber polarization controller and is injected in the waveguide using an optical fiber. In order to avoid the noise due to light passing above the sample or through the substrate, light is collected on top of the output waveguide as shown in Figure 3. This is possible due to a small step fabricated on the edge of the sample that deflects a part of the output light. Moreover, since the waveguide is very thin, the output beam is highly divergent and more light can be collected using a system composed of a long focal length microscope objective (Leica $\times 20, N A=0.4)$ and a highly sensitive InGaAs infra-red camera (Polytec SU 320 $M S-1.7 R T)$. The intensity of light emerging from the cavity can be measured as a function of the wavelength.

Three-dimensional simulations have been performed using a commercial FDTD software (CST Microwave Studio). The red curve in Figure 4 shows the theoretical spectrum. One can observe a transmission peak centered at $\lambda=1501 \mathrm{~nm}$, having a 


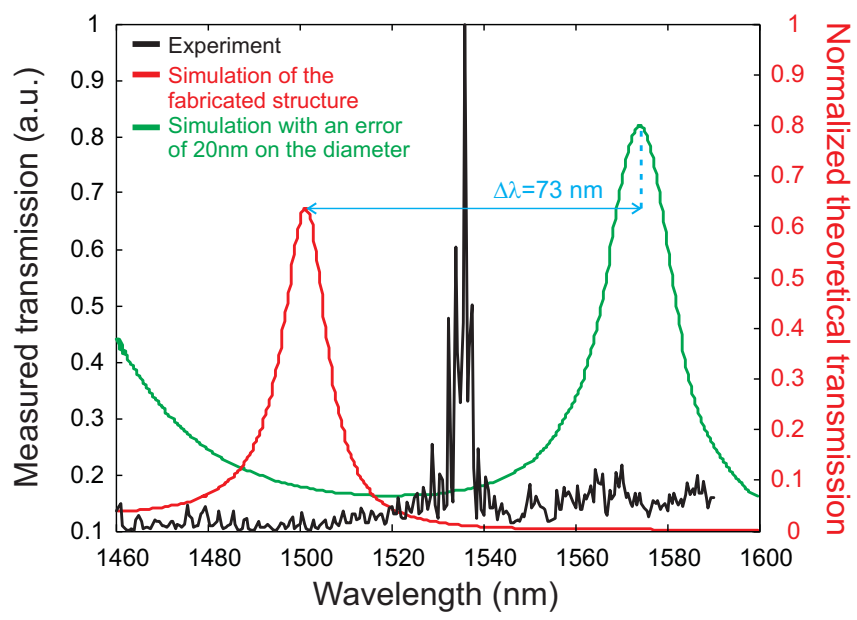

FIG. 4 Comparison between experimental measurements (black curve) and simulation results (red curve) of the transmission through the nanocavity without liquid crystal (type 1). The green curve is the simulation of the cavity with an error of $20 \mathrm{~nm}$ in the diameter.

full width at half maximum of FWHM $=8 \mathrm{~nm}$ and a maximum intensity of $I_{\max } \simeq 63 \%$ of the incident light. The black curve represents the experimental measurements. Note that the two scales are different and cannot be directly compared. Nevertheless, one can observe the presence of a transmission peak centered at $\lambda=1536 \mathrm{~nm}$. This peak is narrower than the predicted one. This is due to the low signal to noise ratio that allows to distinguish only the higher intensity of the transmission peak. Indeed, the bottom of the peak is below the noise level and its shape is modified.

Moreover a shift of $\Delta \lambda=35 \mathrm{~nm}$ can be observed between the theoretical and the experimental peak. This can be explained by a small deviation in the diameter of the holes of the nanocavity. The green curve is the simulation of the same cavity where the sizes of the holes of the nanocavity have been changed by less than $2 \%(20 \mathrm{~nm})$, which is close to the resolution limit of e-beam lithography. A displacement of the peak of $\Delta \lambda \simeq 70 \mathrm{~nm}$ is obtained. The difference between the two peaks in Figure 4 can be attributed to fabrication errors in the order of $10 \mathrm{~nm}$.

\section{SWITCHABLE NANOCAVITY MEASUREMENTS}

The cavity of Type 1 has been replaced by the cavity of type 2 and the liquid crystal has been infiltrated inside the structure. Using the same experimental setup (Figure 3), we have performed transmission measurements at the two temperatures corresponding to the two states of the LC. The results are presented in Figure 5. The measured displacement of the peak is $\Delta \lambda=13 \mathrm{~nm}$.

In this experiment, two principal criteria are important and have an impact on the response of the device to temperature variations. The first one is the orientation of liquid crystals in the holes and at the surface of the sample. This orientation unfortunately cannot be verified.
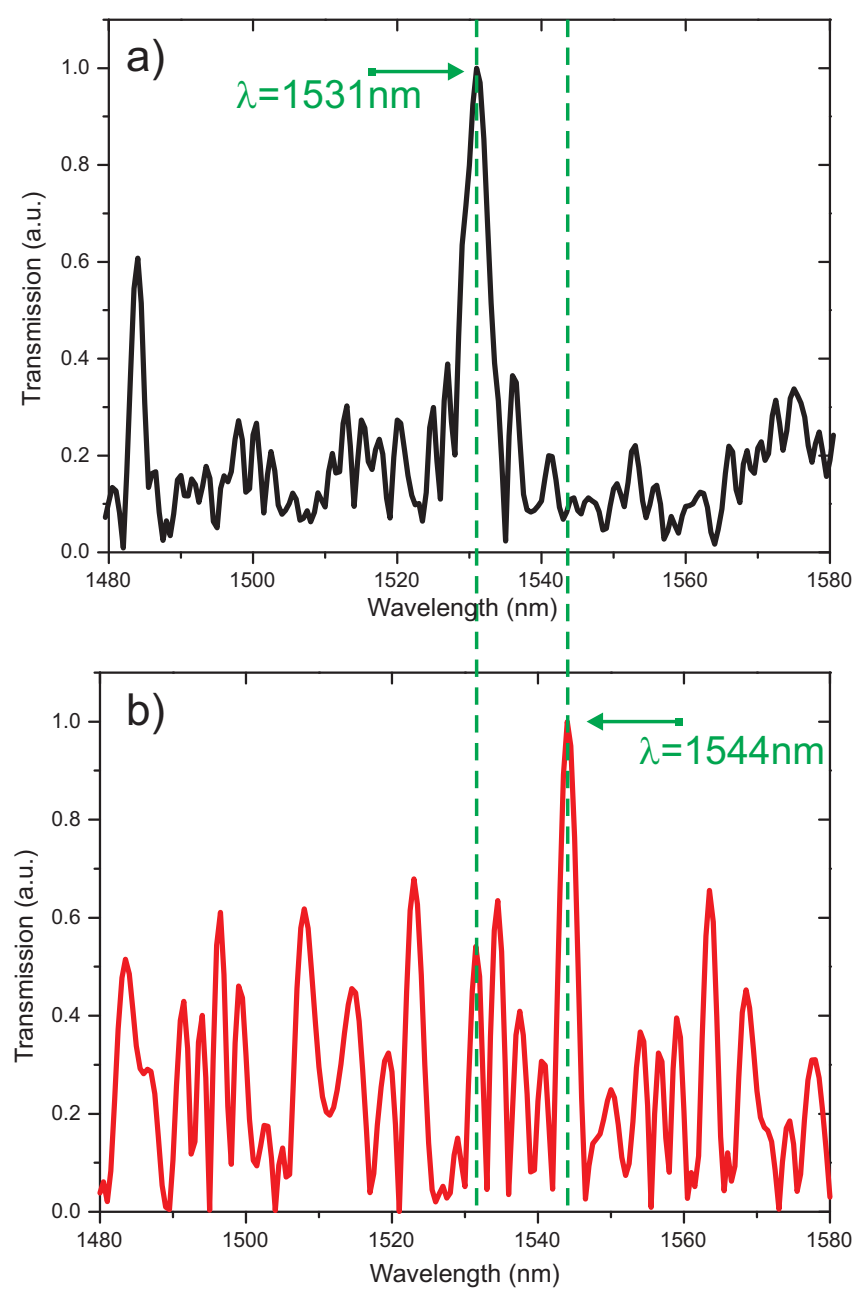

FIG. 5 Experimental measurements of the response in transmission of the nanocavity (type 2 ) infiltrated by liquid crystals. The black curve (a) corresponds to a temperature of $45^{\circ} \mathrm{C}$ (isotropic LC), the red curve (b) corresponds to the room temperature (LC'S are orientated).

The second important parameter in this study is the liquid crystal infiltration inside the nanocavity holes. In order to define the influence of the presence or absence of liquid crystal, we have performed FDTD calculations for three different situations. The first one is the ideal case, where liquid crystal fills the cavity and also the region below and above the membrane (Figure 6(a)). The second case considers only liquid crystal on top and below the cavity, but not inside the holes (Figure 6(b)). Finally, the third example is the non-infiltration case where liquid crystal is only found above the cavity (Figure 6(c)).

The parameters used for modeling the liquid crystals consist in the index of refraction at two temperatures. For the room temperature, the liquid crystal is in planar orientation and the electromagnetic field propagation depends mostly on the extraordinary index $\left(n_{e}=1.68\right.$ at $\left.\lambda=1.55 \mu \mathrm{m}\right)$. To obtain the isotropic phase of the liquid crystal, it has to be heated above $T_{c}=35.3^{\circ} \mathrm{C}$. In our experiment the temperature is set at $T=45^{\circ} \mathrm{C}$, and the index of refraction of the isotropic phase is assumed to be $n_{i}=1.57[19,20]$.

Figure 6 presents for these conditions three graphs with the response of the cavity at room temperature (red curve) and at $T=45^{\circ} \mathrm{C}$ (black curve). At room temperature the extraordinary refractive index is $n_{e}=1.68$ and at $T=45^{\circ} \mathrm{C}, n_{i}=1.57$. 

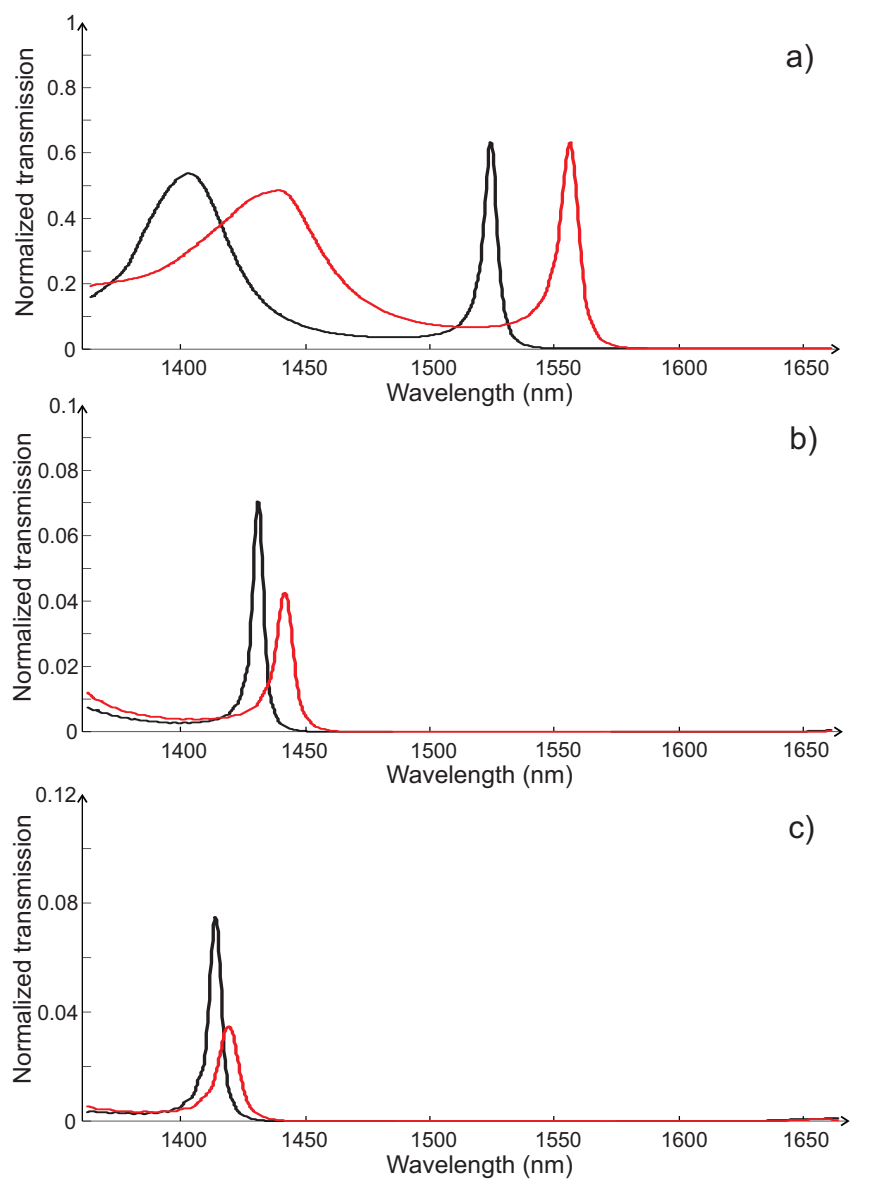

FIG. 6 Calculated variation of the response of the nanocavity in function of the infiltration of liquid crystals at room temperature (red curve, $n=n_{e}=1.68$ ) and at $T=45^{\circ} \mathrm{C}$ (black curve, $n=n_{i}=1.57$ ): (a) liquid crystals infiltrated around and in the nanocavity, (b) liquid crystals above and below the nanocavity but not in the holes, (c) liquid crystal only above (no infiltration of the nanocavity).

Three principal effects are observed (Figure 6) when the infiltration of liquid crystals decreases. First, the maximal amplitude of the peaks decreases drastically. Second, the photonic band gap and the transmission peak shift to the blue and consequently the peak changes position inside the photonic band gap. Third, the shift between the two peaks corresponding to the state of the liquid crystals decreases from $32 \mathrm{~nm}$ (complete infiltration of liquid crystals) to $8 \mathrm{~nm}$ (liquid crystals only at the surface of the structure). From the simulations, it is evident that the LC is not completely infiltrated in the photonic crystal device. One possible cause is surface tension, which prevents the LC to fully infiltrate the holes. This can be improved by performing infiltration of the LC in a vacuum chamber.

\section{CONCLUSION AND DISCUSSION}

We have demonstrated that the response of a filter based on a photonic crystal nanocavity can be tuned using the different phases of liquid crystals. The importance of a good infiltration of the liquid crystals inside the cavity has also been shown. In this paper, the particular behavior of the liquid crystal with the temperature variations has been used to make the nanocavity tunable. In future work, the sample will be activated using electric strains, which can give faster index changes and consequently a higher modulation frequency.

\section{References}

[1] E. Yablonovitch, "Inhibited spontaneous emission in solid-state physics and electronics" Phys. Rev. Lett. 58, 2059-2062 (1987).

[2] S. John, "Strong localization of photons in certain disordered dielectric superlattices" Phys. Rev. Lett. 58, 2486-2489 (1987).

[3] M. Qiu, M. Mulot, M. Swillo, S. Anand, B. Jaskorzynak, A. Karlsson, M. Kamp, and A. Forchel, "Photonic crystal optical filter based on contra-directional waveguide coupling" Appl. Phys. Lett. 83, 51215123 (2003).

[4] L. Fekete, F. Kadlec, P. Kuzel, and H. Nemec, "Ultrafast optoterahertz photonic crystal modulator" Opt. Lett. 32, 680-682 (2007).

[5] L. Gu, W. Jiang, X. Chen, L. Wang, and R. T. Chen, "High speed silicon photonic crystal waveguide modulator for low voltage operation" Appl. Phys. Lett. 90, 071105 (2007).

[6] Y. Jiang, W. Jiang, L. Gu, X. Chen, and R. T. Chen, “80-micron interaction length silicon photonic crystal waveguide modulator" Appl. Phys. Lett. 87, 221105 (2005).

[7] D. Beggs, T. White, L. Cairns, L. O'Faolain, and T. Krauss, “Demonstration of an integrated optical switch in a silicon photonic crystal directional coupler" Physica E 41, 1111-1114 (2009).

[8] H. Kosaka, T. Kawashima, A. Tomita, M. Notomi, T. Tamamura, T. Sato, and S. Kawakami, "Superprism phenomena in photonic crystals" Phys. Rev. B 58, R10096 (1998).

[9] J. Amet, F. Baida, G. Burr, and M.-P. Bernal, "The superprism effect in lithium niobate photonic crystals for ultra-fast, ultra-compact electro-optical switching" Photonic. Nanostruct. 6, 47-59 (2008).

[10] S. Diziain, J. Amet, F. I. Baida, and M.-P. Bernal, "Optical far-field and near-field observations of the strong angular dispersion in a lithium niobate photonic crystal superprism designed for double (passive and active) demultiplexer applications" Appl. Phys. Lett. 93, 261103 (2008).

[11] M. M. de Lima Jr, and P. V. Santos, "Modulation of photonic structures by surface acoustic waves" Rep. Prog. Phys. 68, 1639-1701 (2005).

[12] M. Roussey, F. I. Baida, and M.-P. Bernal, "Experimental and theoretical observations of the slow-light effect on a tunable photonic crystal" J. 0pt. Soc. Am. A 24, 1416-1422 (2007).

[13] E. Camargo, H. Chong, and R. De La Rue, "2D photonic crystal thermo-optic switch based on AlGaAs/GaAs epitaxial structure" Opt. Express 12, 588-592 (2004).

[14] H. H. J. E. Kicken, P. F. A. Alkemade, R. W. van der Heijden, F. Karouta, R. Nötzel, E. van der Drift, and H. W. M. Salemink, "Wavelength tuning of planar photoniccrystals by local processing of individualholes" Opt. Express 17, 22005-22011 (2009).

[15] H.-S. Kitzerow, A. Lorenz, and H. Matthias, "Tuneable photonic crystals obtained by liquid crystal infiltration" Phys. Status Solidi A 204, 3754-3767 (2007).

[16] H. Graener, A. Abdolvand, S. Wackerow, 0. Kiriyenko, and W. Hergert, "Optical properties of photonic/plasmonic structures in nanocomposite glass" Phys. Status Solidi A 204, 3838-3847 (2007).

[17] J. Martz, R. Ferrini, F. Nüesch, L. Zuppiroli, B. Wild, L. Dunbar, R. Houdré, M. Mulot, and S. Anand, "Liquid crystal infiltration of InP-based planar photonic crystals" J. Appl. Phys. 99, 103105 (2006).

[18] K. Takatoh, M. Hasegawa, M. Koden, N. Itoh, R. Hasegawa, and M. Sakamoto, Alignment technologies and applications of liquid crystal devices (Taylor it Francis, London, 2005).

[19] R.-P. Pan, C.-F. Hsieh, C.-L. Pan, and C.-Y. Chen, “Temperature- 
dependent optical constants and birefringence of nematic liquid crystal 5 CB in the terahertz frequency range" J. Appl. Phys. 103, 093523 (2008).
[20] T. Scharf, Polarized light in liquid crystals and polymers (Wiley Interscience, USA, 2006) 